\title{
Un método para la evaluación de riesgos para la salud en zonas mineras
}

Jesús Mejía, M.C., (1) Leticia C arrizales, Q .F.B., ${ }^{(1)}$ Verónica M. Rodríguez, M.C., ${ }^{(1)}$ María E. Jiménez-C apdeville, D r. en $C$., ${ }^{(1)}$ Fernando Díaz-Barriga, D r. en $C$.(1)

\section{Mejía J, Carrizales L, Rodríguez VM, Jiménez-Capdeville ME, Díaz-Barriga F. Un método para la evaluación de riesgos para la salud en zonas mineras. Salud Publica Mex 1999;41 supl 2:S132-S140.}

\section{Resumen}

Objetivo. En este trabajo se propone un esquema de tres fases para evaluar el riesgo en salud en las zonas mineras. La validación del esquema se efectuó en una zona de San Luis Potosí, México. Material y métodos. El monitoreo incluyó análisis de metales en suelo superficial, polvo residencial y agua de pozos. La biodisponibilidad se midió como el nivel de arsénico urinario en niños de las áreas contaminadas. La evaluación toxicológica consistió en análisis de la hepatotoxicidad (niveles de aspartato transaminasa) y neurotoxicidad (niveles de dopamina y de sus metabolitos) en ratas tratadas con residuos mineros. Resultados. Se encontró contaminación por arsénico y plomo en suelo y polvo. La biodisponibilidad fue positiva: $71 \%$ de los niños analizados tuvieron niveles urinarios de arsénico por arriba del valor normal. Los estudios de toxicidad mostraron daño hepático y alteraciones neuro químicas en las ratas tratadas con el residuo. Conclusiones. El método fue útil para demostrar el riesgo en salud y para que las auto ridades decidieran instrumentar un programa de restauración en la zona.

Palabras clave: arsénico; plomo; minería; residuos peligrosos; México

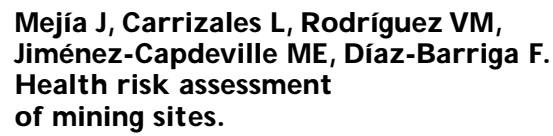

Mejía J, Carrizales L, Rodríguez VM, Jiménez-Capdeville ME, Díaz-Barriga F. Health risk assessment

of mining sites.

Salud Publica Mex 1999;41 suppl 2:S132-S140.

\section{A bstract}

Objective. Considering the health risk associated with mining areas, in this work a methodology for the health assessment of this kind of hazardous sites is proposed. Material and methods. The methodo logy includes a toxicological assessment, an environmental monitoring of metals, and the exposure assessment of the high risk po pulation. The scheme was evaluated in the mining area of Villa de la Paz, San Luis Potosi, Mexico. The toxicological studies were done in rats treated with mining waste, biomarkers of effect for liver and central nervous tissue were analyzed. Metals levels in surface soil, household dust and water were studied. Finally, urinary arsenic was quantified in children. Results. N euro toxicity and hepatotoxicity of the mining waste were shown in rats. Then, arsenic and lead levels were analized in surface soil, household dust, and water. In all three media, exposure po ints, heavily contaminated with both metals, were localized. Finally, high levels of urinary arsenic were fo und in children living in the vicinity of the mine. Conclusions. Taking into account all these results, the Mexican authorities concluded that a high health risk is present in Villa de la Paz, and a remediation program is in progress.

Key words: arsenic; lead; mining; hazardous waste; Mexico
M éxico se ha caracterizado por ser una potencia minera. En la actualidad se encuentra en los primeros lugares del mundo en la producción de arsénico, cadmio, plomo, mercurio, fluorita, bario, etcé- tera. ${ }^{1}$ La minería ha llegado a convertirse en una actividad de gran importancia en algunos estados de la República mexicana, como Guanajuato, Sonora, Chihuahua, Durango, Zacatecas, San Luis Potosí e

(1) Facultad de Medicina, Universidad Autónoma de San Luis Potosí, San Luis Potosí, México.

Fecha de recibido: 13 de mayo de 1998 - Fecha de aprobado: 5 de marzo de 1999

Solicitud de sobretiros: Dr. Fernando Díaz-Barriga, Laboratorio de Toxicología A mbiental, Facultad de Medicina, Universidad Autónoma de San Luis Potosí. Venustiano Carranza 2405, San Luis Potosí, 78210 San Luis Potosí, México. Correo electrónico: rpe7ø82@ pasteur.fmed.uaslp.mx 
Hidalgo. Por consiguiente, los sitios mineros con sus consecuentes exposiciones químicas abundan y su impacto en salud merece ser analizado.

En un sitio minero, dos son las principales fuentes contaminantes: el proceso primario de tratamiento de metales, y la generación de residuos mineros (jales). $\mathrm{Al}$ respecto, cabe mencionar que la minería genera 65\% de los residuos industriales que se producen en México. ${ }^{2}$ En un sitio minero, las principales rutas de exposición pueden ser el material particulado en el aire; el suelo contaminado (por el polvo generado desde la mina, por el material proveniente de los jales o por la deposición del material particulado del aire); el polvo contaminado e inclusive, dependiendo del área geográfica, la contaminación de cuerpos de agua por el lavado de jales o de suelos contaminados.

En consecuencia, las zonas mineras deberían ser consideradas como sitios potencialmente peligrosos para la salud, y sería necesario establecer una metodología especial para su estudio que incluyera la evaluación de tres factores: a) la caracterización de la biodisponibilidad de los metales; $b$ ) el estudio de la toxicidad de los residuos, bajo la perspectiva de que su toxicidad dependerá de la interacción de los metales presentes en ellos, y c) el análisis de la contaminación de los medios del ambiente a fin de determinar las rutas de mayor riesgo para la comunidad afectada.

El primer factor que debe establecerse es la biodisponibilidad de los metales del sitio minero. Este es un punto muy importante, debido a que en la literatura se ha demostrado que los metales cuyo origen es la minería ${ }^{3-5}$ tienen una baja capacidad de absorción. Así, los metales que no pueden absorberse porque forman cristales minerales de baja biodisponibilidad, deben ser considerados de bajo riesgo y el sitio minero de su procedencia, en consecuencia, no merecería ser restaurado. En la literatura se han empleado modelos experimentales para la evaluación de la biodisponibilidad de suelos mineros. ${ }^{5,6}$ No obstante, en este trabajo se ha optado por presentar como alternativa el estudio de biomarcadores de exposición en niños. Esta alternativa tiene dos ventajas: por un lado, permite conocer la biodisponibilidad (una mayor absorción estaría correlacionada con mayores niveles de metales en los tejidos humanos); y, por el otro, permite establecer, de manera simultánea, el nivel de exposición real (si los niños entran en contacto con los medios del ambiente contaminados, tendrían mayores concentraciones corporales de metales).

Ahora bien, la biodisponibilidad de los metales no necesariamente está relacionada con su toxicidad. En los sitios mineros los metales se presentan en mezclas, de tal manera que podría darse el caso de una interacción toxicológica entre ellos. Así por ejemplo, una alta concentración de zinc podría neutralizar la toxicidad del cadmio. ${ }^{7}$ Debe quedar claro que la toxicidad real de un material minero no puede establecerse a través de la caracterización química. Las interacciones de mezclas complejas constituyen una de las limitantes más serias en su conocimiento, en la toxicología moderna.

Para la legislación mexicana, la toxicidad es un factor que se considera proporcional a la cantidad de metal extraído por una solución de ácido acético. ${ }^{*}$ Así, cuanto más es el metal extraído por la solución acética, más tóxico es considerado el material. Por supuesto que este parámetro es bastante limitado, lo que menos toma en cuenta es la posibilidad de la interacción toxicológica entre los metales. A manera de alternativa, la propuesta que se analiza en este trabajo incluye la evaluación toxicológica utilizando un modelo animal. Esta opción ofrece la ventaja de que se puede establecer con precisión la toxicidad total, esto es, la toxicidad dada por los metales presentes en el material que se analiza.

Finalmente, los metales deben ser cuantificados en los diferentes medios del ambiente y en los residuos mineros, cuando los hubiere. Los objetivos de este monitoreo son establecer la magnitud de la contaminación, definir los medios ambientales más impactados por los metales y, por último, identificar los metales que estuvieren en mayor concentración. Los datos facilitan la selección de los biomarcadores de exposición que deberán estudiarse entre la comunidad expuesta, determinan el medio del ambiente cuya toxicidad ameritaría establecerse utilizando el modelo animal, y permiten definir las rutas de exposición que deben atenderse en un programa de restauración.

Por otro lado, la biodisponibilidad, el conocimiento toxicológico y el monitoreo ambiental son factores complementarios entre sí que permiten calificar el riesgo para la salud que significa un sitio minero. Para un funcionario de salud pública, lo anterior implica la reducción de la incertidumbre al momento de decidir sobre la necesidad de aplicar un programa de restauración. Si se toma en cuenta el alto costo económico

\footnotetext{
* Norma Oficial Mexicana (NOM-CRP-001 ECOL/93) que establece las características de los residuos peligrosos, el listado de los mismos y los límites que hacen a un residuo peligroso por su toxicidad al ambiente. Diario Oficial, Viernes 22 de octubre de 1993;2-30; y Norma Oficial Mexicana (NOM-CRP-002 ECOL/93) que establece el procedimiento para llevar a cabo la prueba de extracción para determinar los constituyentes que hacen a un residuo peligroso por su toxicidad al ambiente. Diario Oficial, Viernes 22 de octubre de 1993;31-41.
} 
que implica la limpieza ambiental, el método que se presenta en este trabajo no sólo tiene un valor científico, sino también uno estratégico con respecto al diseño de las políticas ambientales para sitios contaminados.

Debe apuntarse que la presente metodología ya se aplicó, con éxito, en un sitio minero cuyo riesgo para la salud estaba en debate. Los resultados de ese estudio se presentan en este trabajo, y su aplicabilidad en otros sitios se discute en la última sección del documento.

\section{Material y métodos}

\section{Zona de estudio}

De acuerdo con la monografía minera del estado potosino, ${ }^{8}$ en el municipio de Villa de la Paz, San Luis Potosí, existen tres minas que producen plata, oro, plomo, cobre y zinc. Además, en dos de las minas existe arsenopirita. Los residuos de la actividad minera se han ido acumulando con el paso del tiempo, llegando a formar un gran depósito de material fino. Los residuos se encuentran al aire libre y, por consiguiente, han tenido un impacto sobre el ambiente de diversas maneras. En la época de lluvias el material es transportado, por escurrimientos superficiales, hasta el Arroyo de la Paz, que es intermitente y que, a su vez, transporta el residuo minero más de $15 \mathrm{~km}$ curso abajo, hasta que se introduce al subsuelo a través de fracturas geológicas, de tal manera que es posible que contamine el manto acuífero. En época de estiaje, el sedimento seco del arroyo y el material del depósito son transportados por el viento hasta las zonas agrícolas y las áreas residenciales vecinas. Los análisis ambientales se efectuaron en 1997, y los de biodisponibilidad y toxicidad durante 1997 y el primer semestre de 1998.

\section{Biodisponibilidad. Niveles de arsénico en muestras urinarias de niños}

Con el objetivo de realizar un estudio de los niveles de arsénico en orina en la población infantil, se tomaron muestras urinarias de 112 niños residentes de Villa de la Paz. Esa cifra representa aproximadamente 40\% de los niños de tres a seis años de edad que habitan en el poblado. Los niños tenían, cuando menos, dos años de residencia en la zona. El grupo fue seleccionado al azar, como todos los niños cuyos padres dieron autorización para la colecta de la muestra. Se obtuvo la primera muestra de la mañana en recipientes plásticos libres de metales pesados. Los padres dieron su consentimiento por escrito, y se observaron los procedimientos bioéticos establecidos por la Facultad de Medicina.

\section{Toxicidad. Estudio experimental en rata}

Ratas macho de 350-400 g de peso fueron tratadas de cuatro a ocho semanas con $920 \mathrm{mg} /$ día del residuo minero mezclado en el alimento, según el método previamente reportado. ${ }^{9} \mathrm{~A}$ las mismas ratas se les cuantificó el nivel de arsénico cerebral para definir la exposición y, además, los niveles sanguíneos de la enzima aspartato transaminasa como indicador de daño hepático. En cuanto a la evaluación de neurotoxicidad, se optó por una estrategia neuroquímica. Para los análisis se implantó, bajo anestesia, una sonda de microdiálisis en el cuerpo estriado. Después de un periodo de recuperación de dos días, las concentraciones de dopamina (DA) y sus metabolitos ácido 3,4-dihidroxifenil acético (DOPAC) y ácido homovalínico (HVA) fueron cuantificadas por cromatografía de líquidos (HPLC). Se registraron los niveles basales y los obtenidos bajo estímulo de cloruro de potasio (KCl). Para tal efecto, a cada rata se le tomaron tres muestras basales y tres muestras con $\mathrm{KCl}(60 \mathrm{mM})$, con un lapso entre cada una de 20 minutos. Posteriormente se tomaron cuatro muestras para certificar la recuperación del efecto. En los cuadros I y II se señalan las cantidades de animales estudiados. Los tejidos fueron tratados de acuerdo con el método establecido con anterioridad. ${ }^{9}$

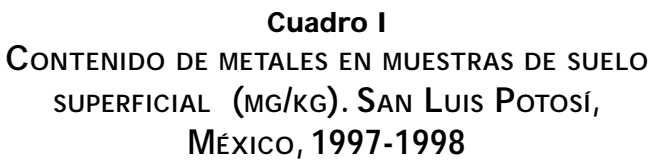

\begin{tabular}{lllrrr} 
Área & Metales & $n$ & Media & DE & Rango \\
Área urbana & Plomo & 9 & 2399 & 1046 & $633-4013$ \\
\hline Villa de la Paz & Arsénico & 9 & 2904 & 2261 & $875-8100$ \\
\hline & Manganeso & 9 & 828 & 262 & $510-1207$ \\
Colonia Real & Plomo & 3 & 309 & 84 & $215-370$ \\
\hline de Minas & Arsénico & 3 & 2390 & 249 & $215-2675$ \\
\hline & Manganeso & 3 & 1002 & 130 & $857-1106$
\end{tabular}

El suelo es externo y superficial. Guías de referencia de laA gencia para las sustancias Tóxicas y el Registro de Enfermedades (ATSD R) para arsénico en suelo $=20 \mathrm{mg} / \mathrm{kg}$ (datos para población infantil) ${ }^{10}$ y para manganeso en suelo $=300 \mathrm{mg} / \mathrm{kg}$ (datos para población infantil) ${ }^{10}$. N ivel recomendado para plomo en suelo en áreas recreativas (población infantil) $=250$ $\mathrm{mg} / \mathrm{kg}^{11}$ 


\section{Cuadro II \\ Contenido de metales en muestras de polvo casero (Mg/Kg). San Luis Potosí, MÉXıco, 1997-1998}

\begin{tabular}{lllrrrr} 
Área & Metales & $n$ & Media & DE & \multicolumn{1}{c}{ Rango } \\
Área urbana & Plomo & 12 & 1824 & 1260 & $555-5190$ \\
\hline Villa de la Paz & Arsénico & 12 & 2045 & 1117 & $352-4050$ \\
\hline & Manganeso & 12 & 619 & 232 & $238-1070$ \\
\hline & & & & & \\
Colonia Real & Plomo & 6 & 417 & 221 & $220-778$ \\
\hline de Minas & Arsénico & 6 & 4960 & 3318 & $1780-9950$ \\
\hline & Manganeso & 6 & 928 & 185 & $780-1250$
\end{tabular}

\section{Toxicidad. Extracción con ácidos}

La extracción con ácido acético se hizo siguiendo el método considerado en la legislación mexicana. ${ }^{*} \mathrm{Se}$ pusieron en contacto $10 \mathrm{~g}$ de residuo minero con 200 $\mathrm{ml}$ de una solución de ácido acético $0.1 \mathrm{~N}$. La mezcla se agitó durante 18 horas a temperatura ambiente. $\mathrm{Al}$ término, la mezcla se pasó a través de un filtro de fibra de vidrio de 0.6-0.8 $\mu \mathrm{m}$ de diámetro. Posteriormente, la solución extraída se digirió con una mezcla de ácido nítrico/ácido perclórico (6:1) para la cuantificacion del arsénico por espectrofotometría de absorción atómica con generador de hidruros. En cuanto a la extracción con ácido clorhídrico $(\mathrm{HCl})$, durante dos horas se pusieron en contacto dos gramos del residuo minero con $50 \mathrm{ml}$ de $\mathrm{HCl} 1 \mathrm{~N}$, a temperatura ambiente. Al término se filtró empleando un filtro de fibra de vidrio de 0.6-0.8 $\mu \mathrm{m}$ de diámetro y el material extraído fue aforado a $100 \mathrm{ml}$ con agua desionizada. La cuantificación se realizó de la misma manera que con el extracto de ácido acético.

\section{Monitoreo ambiental}

El muestreo se hizo de acuerdo con el procedimiento indicado en el Manual de muestreo ambiental, publicado por la Agencia para las Sustancias Tóxicas y el Re-

\footnotetext{
* Norma Oficial Mexicana (NOM-CRP-001 ECOL/93) que establece las características de los residuos peligrosos, el listado de los mismos y los límites que hacen a un residuo peligroso por su toxicidad al ambiente. Diario Oficial, Viernes 22 de octubre de 1993;2-30; y Norma Oficial Mexicana (NOM-CRP-002 ECOL/93) que establece el procedimiento para llevar a cabo la prueba de extracción para determinar los constituyentes que hacen a un residuo peligroso por su toxicidad al ambiente. Diario Oficial, Viernes 22 de octubre de 1993;31-41.
}

gistro de Enfermedades (ATSDR). ${ }^{12}$ A continuación se expone el diseño que se siguió en cada uno de los puntos de exposición. Con el fin de seleccionar los puntos de muestreo en suelo superficial, se utilizó un sistema de información geográfica del poblado de Villa de la Paz. Se recolectaron muestras tanto en el área urbana del poblado, que está cercana a la planta trituradora de la mina, como en una de las colonias periféricas, la colonia Real de Minas, situada en proximidad al depósito de residuos. Las muestras de polvo residencial se recolectaron en puntos cercanos a las zonas de muestreo de suelo superficial. En el Arroyo de la Paz se tomó una muestra superficial del sedimento. El ejido La Carbonera, que fue afectado por desbordamientos del Arroyo de la Paz, y la zona agrícola de Cerrito Blanco, que es regada con aguas ricas en arsénico, también quedaron incluidos en el análisis de metales en suelo. Para agua, se colectaron muestras de pozos tanto cercanos como lejanos al área minera. El polvo se recolectó del interior de las casas, "barriendo" con brochas limpias las áreas más transitadas por los infantes de la vivienda. El suelo se tomó de la superficie, utilizando espátulas cuya limpieza fue previamente certificada. Las muestras de agua se colectaron en recipientes plásticos lavados con ácido y agua desionizada. Estas muestras fueron acidificadas al momento de la recolección, y transportadas al laboratorio bajo refrigeración.

\section{Cuantificación de metales}

Los metales fueron cuantificados por espectrofotometría de absorción atómica, con generador de hidruros para arsénico y con horno de grafito para plomo, cobre y manganeso. Las muestras de agua fueron digeridas con una mezcla de nítrico-perclórico (3.0:0.5), y las de suelo y polvo con horno de microondas, utilizando nítrico al $25 \%$. Como control de calidad se efectuaron análisis de estándares certificados. Para suelo se utilizó el estándar NIST-SRM 2710 ("Montana soil I") y se logró una recuperación superior a 90\% en todos los metales. Para agua se utilizó un estándar de la Agencia para la Protección Ambiental (EPA, por sus siglas en inglés), con una recuperación superior a 95\%. En el caso de arsénico en orina, el metal también fue cuantificado por espectrofotometría de absorción atómica con generador de hidruros. Las muestras de orina fueron digeridas con una mezcla de nítrico-perclórico-sulfúrico $(3 \mathrm{ml} / 0.5 \mathrm{ml} / 0.5 \mathrm{ml})$. Como control de calidad se efectuaron análisis de un estándar certificado NIST-SRM 2670 ("Toxic Metals in Freeze-Dried Urine"), y se logró una recuperación de $102 \%$. 


\section{Resultados}

\section{Monitoreo ambiental}

En primer término, se estudió el contenido de diferentes metales en muestras del residuo minero de Villa de la Paz, San Luis Potosí. Los niveles de arsénico se registraron a $9647 \mathrm{mg} / \mathrm{kg}$, los de manganeso a 1650 $\mathrm{mg} / \mathrm{kg}$, los de cobre a $1180 \mathrm{mg} / \mathrm{kg}$ y los de plomo a $690 \mathrm{mg} / \mathrm{kg}$. Tomando en cuenta estas concentraciones y su toxicidad reportada en la literatura, el arsénico, el manganeso y el plomo fueron elegidos como contaminantes críticos y se determinaron sus niveles en suelo superficial y en polvo residencial. En los cuadros I y II se presentan los resultados del monitoreo efectuado tanto en el área urbana de Villa de la Paz como en la colonia Real de Minas (zona próxima al depósito de los residuos mineros).

Considerando los niveles promedio en suelo superficial que aparecen en el cuadro I, se definió que para el área urbana de Villa de la Paz el arsénico se ubicó 145 veces por arriba de su valor de referencia; el plomo registró niveles casi 10 veces superiores al valor de referencia, y el manganeso solamente estuvo dos veces por arriba de su guía ambiental. En comparación, los valores de la colonia Real de Minas se mantuvieron similares a los del área urbana en cuanto a manganeso y arsénico pero, sorpresivamente, las concentraciones de plomo disminuyeron de manera considerable hasta ubicarse en el rango de su valor de referencia.

Por otra parte, las concentraciones promedio de metales en el polvo recolectado al interior de las viviendas, presentaron, en general, la distribución observada para suelo (cuadro II), esto es, altos valores de plomo en el área urbana de Villa de la Paz y bajos en la colonia Real de Minas. Por su parte, el arsénico y el manganeso se mantuvieron constantes en ambas áreas. Los tres metales presentaron concentraciones similares tanto en suelo superficial (cuadro I) como en polvo residencial (cuadro II). Quizá podría observarse alguna diferencia respecto al arsénico en la colonia Real de Minas pero, en este caso, los mayores niveles registrados en polvo se debieron a que hubo una vivienda con una concentración extrema de 9950 $\mathrm{mg} / \mathrm{kg}$ de arsénico en polvo, lo cual modificó el nivel promedio.

Los resultados en suelo y polvo pueden explicarse al tomar en cuenta que el área de la mina tiene dos fuentes importantes de contaminación: la planta de trituración, que cuenta con una quebradora de mineral crudo, y el depósito de residuos mineros. La primera sería fuente contaminante para el área urbana de Villa de la Paz, y el depósito sería el origen de la contaminación para la colonia Real de Minas. El mineral crudo tratado por la quebradora de la planta de trituración es rico en plomo, manganeso y arsénico; empero, precisamente debido a que la planta concentra plomo, los residuos contienen concentraciones menores de este metal. En consecuencia, habría más plomo en la zona urbana y menos en la colonia Real de Minas.

Considerando que los residuos mineros podrían ser transportados por los escurrimientos que se presentan en la época de lluvias, se cuantificaron los niveles de arsénico y plomo en suelo superficial a lo largo del trayecto del Arroyo de la Paz. Así, se localizaron mayores concentraciones de arsénico y de plomo en las cercanías del depósito (2 $159 \mathrm{mg} / \mathrm{kg}$ de arsénico y $1012 \mathrm{mg} / \mathrm{kg}$ de plomo), en el curso del Arroyo de la $\mathrm{Paz}$ (7 $102 \mathrm{mg} / \mathrm{kg}$ de arsénico y $464 \mathrm{mg} / \mathrm{kg}$ de plomo), y en el suelo superficial del ejido La Carbonera (4 087 $\mathrm{mg} / \mathrm{kg}$ de arsénico y $691 \mathrm{mg} / \mathrm{kg}$ de plomo). Estos niveles son sumamente elevados si se comparan con los niveles basales de la región ( $45 \mathrm{mg} / \mathrm{kg}$ de arsénico y 3 $\mathrm{mg} / \mathrm{kg}$ de plomo).

Se siguió el mismo monitoreo piloto para investigar los niveles del agua subterránea en pozos cercanos al depósito. Los resultados preliminares indican que los niveles de arsénico en los dos pozos cercanos al depósito fueron mayores a los registrados en pozos más alejados. Por ejemplo, los pozos de La Florida y del Hotel, cercanos al depósito, tuvieron niveles de arsénico de $106 \mu \mathrm{g} / 1$ y de $6897 \mu \mathrm{g} / 1$, respectivamente. En tanto, los pozos en el área control a $30 \mathrm{~km}$ del área y presumiblemente ubicados en otro acuífero, tuvieron concentraciones de 20 y $14 \mu \mathrm{g} / 1$ de arsénico en agua. Los datos deben ser confirmados en futuros monitoreos, pero indican una grave contaminación que debe atenderse de inmediato.

\section{Biodisponibilidad}

Debido a la alta concentración de arsénico en los distintos medios ambientales evaluados en ambas regiones (área urbana y colonia Real de Minas), se decidió efectuar un estudio. Sus objetivos prinicipales eran establecer la biodisponibilidad del arsénico y definir la magnitud de la exposición entre los niños. Si el arsénico no fuere biodisponible, o no hubiere exposición, la cantidad de arsénico urinario sería baja y, por lo tanto, los niveles ambientales no representarían un riesgo para la salud. Sin embargo, los datos del cuadro III indican que $71 \%$ de los niños analizados tuvieron niveles de arsénico en orina por arriba del valor normal (50 $\mu \mathrm{g} /$ gr creatinina) y $28 \%$ los tuvieron por arriba del doble del valor normal. Estos porcentajes fueron 


\section{Cuadro III \\ Niveles de arsénico en orina en niños de la villa de la paz ( $\mu \mathrm{g} / \mathrm{g}$ creatinina). San Luis Potosí, México, 1997-1998}

\begin{tabular}{lcccccc} 
& $\mathrm{n}$ & Media & $\mathrm{DE}$ & Rango & Porcentaje $>50 \mu \mathrm{g} / \mathrm{gc} *$ & Porcentaje $>100 \mu \mathrm{g} / \mathrm{gc*}$ \\
Área urbana de Villa de la Paz & 62 & 76.2 & 2.0 & $18.8-497.7$ & 87 & 66 \\
\hline Colonia Real de Minas & 50 & 63.9 & 1.9 & $17.7-248.7$ & 31 & 24 \\
\hline Total & 112 & 70.5 & 1.9 & $17.7-497.7$ & 71 & 28
\end{tabular}

$\mathrm{n}$ (número de niños estudiados). Media (media aritmética). D.E. (desviación estándar). $\mu \mathrm{g} / \mathrm{gc}$ ( $\mu \mathrm{g} / \mathrm{g}$ creatinina). Total (los datos incluyen a todos los niños). $\mathrm{N}$ ivel de acción para arsénico en orina para niños no expuestos : menor a $50 \mu \mathrm{g} / \mathrm{g}$ creatinina.* $p<0.05^{13}$

superiores en el área urbana, lo cual podría suponer una ruta de exposición no explorada en este trabajo, como por ejemplo la inhalación o ingesta de material particulado proveniente de la quebradora. En conclusión, los datos permiten definir que el arsénico es biodisponible y que los niños están expuestos al mismo, tanto en la colonia Real de Minas como en el área urbana.

\section{Toxicidad}

En este trabajo se informa acerca de los estudios efectuados sobre el material depositado como residuo minero; los análisis sobre la toxicidad del suelo contaminado están todavía en proceso. Para evaluar la toxicidad se han seguido dos procedimientos: el establecido por la ley mexicana para definir la toxicidad de un residuo sólido, ${ }^{*}$ y la evaluación toxicológica empleando un modelo animal. Con la primera estrategia se encontró que los niveles de arsénico en un extracto de ácido acético del residuo, no rebasaron el límite de los $5 \mathrm{mg} / 1$ con el cual la norma mexicana define a un residuo como tóxico. No obstante, debe quedar claro que en los seres vivos el ácido que podría extraer el metal del residuo no es el acético, sino el $\mathrm{HCl}$ que se encuentra presente en el tracto digestivo. Por lo tanto, también se realizó la extracción con este ácido. El resultado fue que, con nueve horas menos de extracción que el ácido acético, el $\mathrm{HCl}$ extrajo 67 veces más arsénico y los valores así obtenidos sí superaron la norma de los $5 \mathrm{mg} / \mathrm{l}$.

\footnotetext{
* Norma Oficial Mexicana (NOM-CRP-001 ECOL/93) que establece las características de los residuos peligrosos, el listado de los mismos y los límites que hacen a un residuo peligroso por su toxicidad al ambiente. Diario Oficial, Viernes 22 de octubre de 1993;2-30; y Norma Oficial Mexicana (NOM-CRP-002 ECOL/93) que establece el procedimiento para llevar a cabo la prueba de extracción para determinar los constituyentes que hacen a un residuo peligroso por su toxicidad al ambiente. Diario Oficial, Viernes 22 de octubre de 1993;31-41.
}

\author{
Cuadro IV \\ Niveles sanguíneos de aspartato transaminasa \\ en ratas adultas. San Luis Potosí, \\ México, 1997-1998 \\ Concentración $\quad 9.9 \pm 2.2 \quad 35.5 \pm 14.9 * \quad 33.8 \pm 0.4 * *$
}

Los valores representan la media \pm error estándar ( $n=4$ a 6 animales). Los asteriscos indican diferencias significativas con respecto al control (Tukey HSD); $* p<0.01 ; * *<0.05$. Las concentraciones se dan en UI / I

\section{Cuadro V \\ Concentración de dopamina y sus metabolitos EN MICRODIALISADOS DE CEREBRO DE RATAS ADULTAS. San Luis Potosí, Méxıco, 1997-1998}

\begin{tabular}{lrrrr} 
& Control & $\begin{array}{c}\text { Cuatro } \\
\text { semanas }\end{array}$ & $\begin{array}{c}\text { Ocho } \\
\text { semanas }\end{array}$ \\
Dopamina & Basal Estimulada & $3.4 \pm 0.8$ & $4.3 \pm 0.5$ & $3.6 \pm 0.4$ \\
\hline & $21.7 \pm 5.8$ & $6.4 \pm 1.0^{*}$ & $6.2 \pm 1.0^{*}$ \\
\hline DOPAC & Basal Estimulado & $113.7 \pm 14.3$ & $122.8 \pm 19.5$ & $161.1 \pm 6.3$ \\
\hline & & $114.1 \pm 15.1$ & $92.7 \pm 17.0$ & $109.1 \pm 10.9$ \\
\hline HVA & Basal Estimulado & $88.4 \pm 13.3$ & $33.8 \pm 6.7^{*}$ & $71.2 \pm 4.6$ \\
\hline & & $84.1 \pm 13.3$ & $29.9 \pm 5.2^{* *}$ & $39.1 \pm 6.0^{*}$
\end{tabular}

Los valores representan la media \pm error estándar ( $=3$ a 8 animales). Se promediaron tres muestras en cada condición (basal y estimulada) para cada animal. Los asteriscos indican diferencias significativas con respecto al control respectivo (Tukey HSD); $p<0.05 ;{ }^{* *} p<0.01$. Las ratas estimuladas fueron tratadas con $\mathrm{KCl}$ como se describe en la sección de métodos

Considerando que los datos obtenidos con ambos procedimientos de extracción son insuficientes para declarar tóxico al residuo minero, dado que éstos no son un parámetro toxicológico, se optó por efectuar mayores estudios. Se procedió a utilizar un modelo animal y se administró el residuo por vía oral a ratas. A continuación se evaluaron los efectos hepáticos y 
neuroquímicos; en el primer caso, se determinaron los niveles de la enzima hepática aspartato transaminasa en la sangre de las ratas. Los valores de la enzima fueron superiores en las ratas tratadas con el residuo en comparación con las ratas controles (cuadro IV). En cuanto a la evaluación de neurotoxicidad, se analizó la secreción de la dopamina y sus metabolitos DOPAC y HVA. En el cuadro V puede observarse que, en comparación con las ratas controles, las ratas tratadas con el residuo y bajo estímulo de $\mathrm{KCl}$ presentaron una inhibición de la secreción de la dopamina y su metabolito HVA. Es importante hacer notar que las ratas tratadas durante cuatro semanas tuvieron niveles de arsénico en cerebro 21 veces superiores al nivel que se encontró en las ratas control. Por su parte, las ratas tratadas durante ocho semanas presentaron valores del metaloide 29 veces superiores al control.

\section{Discusión}

Los sitios mineros pueden tener altos índices de contaminación. El mal manejo de los residuos o el escaso control ambiental en los procesos de recuperación de metales podrían ser el origen de esa situación. En las áreas mineras contaminadas, son diversos los medios del ambiente que están impactados por los metales: el aire, el suelo, el polvo residencial, las fuentes de agua potable e, inclusive en algunas zonas, los alimentos. El conocimiento de la concentración de los metales en los diferentes medios, a través del monitoreo, es un gran avance; sin embargo, no basta para definir el riesgo que representan para la salud. En los sitios mineros los metales suelen presentarse en forma de minerales, esto es, asociados a otros elementos. Así, la arsenopirita es un complejo de arsénico con hierro y azufre. La biodisponibilidad y toxicidad de los minerales suelen ser menores a la de los metales libres. Por consiguiente, el análisis de riesgo en una zona minera debe realizarse mediante métodos que integren el monitoreo, la biodisponibilidad y la toxicidad. Estos parámetros, en su conjunto, permiten definir el riesgo para la salud $y$, por consiguiente, facilitan la toma de decisiones al momento de diseñar programas de intervención.

En este trabajo se presentan los resultados de un estudio efectuado en la zona minera de Villa de la Paz. Esta zona había sido tema de debate ya que las quejas de la población se enfrentaban a la decisión de las autoridades de considerar al sitio como una área de bajo riesgo. Tal decisión se fundamentaba, sobre todo, en que los residuos del sitio no eran considerados tóxicos debido a que se encontraban dentro de la norma mexicana que fija la toxicidad de los residuos sólidos. El grupo de investigación que llevó a cabo el presente trabajo aprovechó el caso para probar la metodología que proponía para el análisis de sitios mineros. Así entonces, se realizaron estudios de monitoreo ambiental, de biodisponibilidad y de toxicidad. A continuación se aborda cada uno de ellos.

Los datos del monitoreo ambiental indicaron contaminación en suelo y polvo residencial (cuadros I y II). Además, mediante evaluaciones preliminares también se registró impacto por arsénico en el acuífero de la zona; precisamente, las concentraciones más elevadas fueron las de arsénico. El contenido de plomo en el área urbana fue elevado, pero no así en la colonia Real de Minas. En tanto, el manganeso se registró ligeramente por arriba del valor de referencia en ambas zonas de estudio. Como ya se mencionó, estos resultados sugieren la existencia de dos fuentes contaminantes: para el área urbana, sería la planta quebradora de material crudo que podría estar despidiendo material particulado rico en plomo, arsénico y manganeso; y para la colonia Real de Minas, sería el depósito de residuos mineros, que contiene material rico en arsénico y manganeso. Los residuos tendrían menos plomo que el material particulado, debido a que uno de los procesos de la planta minera es, justamente, la recuperación del plomo.

Con estos datos se desprende que, hasta el momento, existen tres rutas claras de exposición: la ingesta de suelo contaminado tanto en la colonia Real de Minas como en el área urbana; la ingesta de polvo residencial, también en ambas zonas; y, por último, la inhalación o ingesta de material particulado. En el área urbana es clara la presencia del material particulado que desprende la quebradora; pero en la colonia Real de Minas, podría establecerse una ruta similar para el polvo que acarrea el viento, desde el depósito de los residuos hasta la zona habitacional. Tomando en cuenta estas rutas de exposicion y habiendo establecido que los prinicipales metales en el ambiente son el plomo y el arsénico, fue posible definir que los niños son el grupo de mayor riesgo. Los niños son quienes más están en contacto con el suelo y, además, absorben más plomo que los adultos. ${ }^{14}$

Los datos fueron claros y demostraron la existencia de una problemática. Sin embargo, se generaron otras incertidumbres: sobre la biodisponibilidad de los metales encontrados; sobre la real exposición de los niños a dichos metales; $y$, sobre la toxicidad del material contaminado. Con respecto a esta última, las autoridades ya habían declarado que era baja.

A fin de establecer simultáneamente tanto la biodisponibilidad de los metales tóxicos como la exposición infantil a los mismos, se decidió realizar un estudio de biomarcadores de exposición. Se selec- 
cionó al arsénico como el metal crítico y se decidió cuantificar sus concentraciones en orina a manera de biomarcador. Se tomó al arsénico como indicador debido a que fue el metal tóxico más abundante, tanto en el área urbana como en la colonia Real de Minas.

Se postuló que si los metales eran biodisponibles y si los niños entraban en contacto con los medios contaminados, entonces se registrarían altos niveles de arsénico urinario. Los resultados que se presentan en el cuadro III hablan por sí mismos: casi tres cuartas partes de los niños estudiados tuvieron niveles de arsénico en orina superiores a lo normal. Además, en $66 \%$ de los niños del área urbana se encontraron concentraciones mayores al doble de lo normal. Esos datos indicaron que, efectivamente, el arsénico era biodisponible y que los niños estaban expuestos a ambientes contaminados con el mismo. De esta manera podría establecerse el riesgo para la salud. Se había demostrado una correlación entre la contaminación del ambiente por arsénico y la exposición infantil al metaloide. Sin embargo, las autoridades sostenían todavía, que, de acuerdo con sus pruebas, la toxicidad del material era baja.

El señalamiento de las autoridades sobre la toxicidad, específicamente de los residuos, estaba sustentada en la legislación mexicana. La extracción con ácido acético había dado resultados negativos. Cuando los autores del presente trabajo emplearon dicho parámetro, que legalmente es utilizado para validar la toxicidad, ${ }^{*}$ también resultó con datos negativos al tratar a una muestra de los residuos mineros. Para demostrar aún más la invalidez de la extracción como un procedimiento para evaluar toxicidad, se llevó a cabo un segundo procedimiento empleando ácido clorhídrico en lugar de ácido acético. Cuando menos el uso del clorhídrico tiene mayor validez científica si se considera que es el ácido que se encuentra en el aparato digestivo humano. Con este ácido hubo mayor extracción de arsénico que con el ácido acético, lo cual no indicaba una mayor toxicidad. Ninguno de los dos métodos demuestra interacción toxicológica entre los metales presentes en el residuo; tampoco dan evi-

\footnotetext{
* Norma Oficial Mexicana (NOM-CRP-001 ECOL/93) que establece las características de los residuos peligrosos, el listado de los mismos y los límites que hacen a un residuo peligroso por su toxicidad al ambiente. Diario Oficial, Viernes 22 de octubre de 1993;2-30; y Norma Oficial Mexicana (NOM-CRP-002 ECOL/93) que establece el procedimiento para llevar a cabo la prueba de extracción para determinar los constituyentes que hacen a un residuo peligroso por su toxicidad al ambiente. Diario Oficial, Viernes $22 \mathrm{de}$ octubre de 1993;31-41.
}

dencia sobre la distribución de los metales en el organismo y mucho menos aportan información sobre la operatividad de los sistemas de defensa celular o sobre los sistemas de excreción. Debe concluirse, entonces, que la extracción es un procedimiento químico que dista mucho de ser un modelo de evaluación toxicológica.

Sin embargo, este grupo de investigación buscaba demostrar la toxicidad real del residuo minero: se necesitaban pruebas que demostraran la invalidez de la metodología oficial. Se optó por un método alternativo, el uso de animales de laboratorio. Se seleccionaron ratas a las cuales se les administró el residuo por vía oral. Los datos obtenidos indican que el residuos es hepatotóxico y neurotóxico (cuadros IV y V). Estos efectos se explican por los antecedentes de algunos de los metales tóxicos más abundantes en el residuo, como es el caso para plomo, ${ }^{14}$ arsénico ${ }^{15} \mathrm{y}$ manganeso. ${ }^{16}$ Es importante tomar en cuenta que los efectos tóxicos que se encontraron en estas ratas, son el resultado de la mezcla de todos los metales presentes en el residuo (en un estudio preliminar quedó demostrada la presencia, también, de cromo, cobre, níquel y cadmio en este material). En conclusión, el modelo animal es muy superior a la extracción ácida como método para determinar toxicidad, y los datos aquí aportados indican que el residuo minero estudiado es tóxico.

En su conjunto, los datos del monitoreo ambiental, del uso de biomarcadores en niños y del empleo del modelo ambiental, demostraron: contaminación, biodisponibilidad y exposición infantil a un material tóxico. Los datos sirvieron para que la autoridad adoptara una nueva actitud y como resultado se firmó un convenio entre la empresa minera y las autoridades gubernamentales para la restauración del sitio. Por otra parte, se planteó la necesidad de estudiar los posibles efectos sobre la salud que pudieran estar presentándose en la población, y de llevar a cabo el monitoreo de los niveles de arsénico en el acuífero de la región. El método fue útil para disminuir el riesgo a la salud y, por ende, puede utilizarse en otros sitios mineros.

Actualmente se aplica la misma secuencia metodológica en el estudio de otros dos sitios mineros; inclusive, al revisar el estudio de impacto ambiental que una compañía ha presentado para una nueva operación minera, se propuso la inclusión de los aspectos de monitoreo, biodisponibilidad, exposición y toxicidad de los materiales que se generarán como polvos o residuos. Al parecer, en una primera aproximación la compañía ha aceptado emplear tales parámetros para definir el riesgo para la salud. Así, hoy en día se busca que esos parámetros sirvan para la prevención 
del riesgo y no únicamente para validar la restauración de un sitio contaminado.

\section{A gradecimientos}

Este trabajo se realizó gracias al apoyo que brindó el Consejo Nacional de Ciencia y Tecnología, a través del Sistema Miguel Hidalgo (proyecto RN/26 /96).

\section{Referencias}

1.La minería en México. Instituto $\mathrm{N}$ acional de Estadística, Geografía e Informática. México: IN EGI 1996.

2.SEDESO L. Informe de la situación general en materia de equilibrio ecológico y protección al ambiente 1991-1992. Secretaría de Desarrollo Social. México, 1993: 193-206.

3. Ruby MV, D avis A, Schoof R, Eberle S, Sellstone CM. Estimation of lead and arsenic bioavailability using a physiologically based extraction test. Environ Sci Technol 1996; 30: 422-430.

4. Danse IHR, Garb LG, Moore RH. Blood lead surveys of communities in proximity to lead-containing mill tailings. Am Ind Hyg Assoc J 1995; 56: 384-393.

5. Freeman GB, Schoof RA, Ruby MV, Davis AO, Dill JA, Liao SC et al.. Bioavailability of arsenic in soil and house dust impacted by smelter activities following oral administration in Cynomolgus monkeys. Fundam Appl Toxicol 1995: 28:215-222.
6. Freeman GB, Johnson JD, Killinger JM, Liao SC, D avis AO et al. Bioavailability of arsenic in soil impacted by smelter activities following oral administration in rabbits. Fundam A ppl Toxicol 1993; 21: 83-88.

7.A gency for Toxic Substances and Disease Registry. Toxicological profile for cadmium. Atlanta GA:ATSD R U.S. D epartment of Health and Human Services, 1997.

8. Consejo de Recursos Minerales. Monografía geológico-minera del estado de San Luis Potosí. México, D.F.: Secretaría de Energía, Minas e Industria Paraestatal, 1992.

9. Rodríguez VM, D ufour L, C arrizales L, Díaz-Barriga F, Jiménez-C apdeville ME. Effects of oral exposure to mining waste on in vivo dopamine release from rat striatum. Environ Health Perpect 1998;106:487-491.

10. Agency for Toxic Substances and Disease Registry. Soil comparison values. U.S.D epartment of H ealth and H uman Services.Atlanta GA:ATSD R, 1997.

11. Madhavan S, Rosenman KD, Shehata T. Lead in soil: Recommended maximum permissible levels. Environ Res 1989;49:136-142.

12.Agency for Toxic Substances and Disease Registry. Environmental data needed for public health assessments. A guidance manual. U.S. Department of Health and Human Services. Atlanta GA:ATSD R 1994.

13. H wangYH, Bornschein RL, Grote J, Menrath W, Roda S. Environmental arsenic exposure of children around a former copper smelter site. Environ Res 1997:72:72-81.

14. Agency for Toxic Substances and Disease Registry. U.S. Toxicological profile for lead. D epartment of $H$ ealth and Human Services.Atlanta (GA), ATSDR, 1997.

15. Agency for Toxic Substances and Disease Registry. U.S. Toxicological profile for arsenic. Department of Health and Human Services. Atlanta GA:ATSD R, 1993

16. A gency for Toxic Substances and Disease Registry. U.S. Toxicological profile for manganese. D epartment of H ealth and Human Services.A tlanta GA:ATSDR 1997 